\title{
Les enjeux de la mémoire chez les historiennes des femmes, 1970-2001
}

Les féministes ont très tôt formulé l'idée selon laquelle la marginalisation du féminin dans l'histoire participe de la marginalisation des femmes au présent. Ainsi, dès le XIX ${ }^{\mathrm{e}}$ siècle, des militant-e-s de l'égalité, s'appliquent à remédier à ce que Natalie Zemon Davis appelle «les silences patriarcaux du passé $»^{1}$ en conservant des traces de leurs actions, en commémorant des événements et figures du passé et en s'essayant à l'écriture de l'histoire, celle des femmes notamment. Cependant, la discipline historique qui se professionnalise à cette période exclut les historiennes des cercles historiographiques, renvoyées à un prétendu amateurisme féminin ${ }^{2}$. Jusqu'au milieu du $\mathrm{XX}^{\mathrm{e}}$ siècle $^{3}$, cette construction masculine de la discipline historique conduit à une occultation de la dimension sexuée des phénomènes étudiés. En France, à la fin des années 1960, le contexte institutionnel change : au moment même où les mobilisations féministes connaissent un regain d'activité et de visibilité dans l'espace public et stimulent la demande sociale en connaissances sur le passé des femmes, l'université et la discipline historique se transforment. Cette période, marquée par l'avènement de ce qu'on a appelé la «nouvelle histoire »-courant historiographique porté par la troisième génération de l'École des Annales et centré sur l'«histoire des mentalités »-s'avère particulièrement favorable à la construction des femmes comme objet d'études. Dans les années 1970, des enseignements et des groupes de recherche spécialisés dans l'histoire des femmes se créent au sein des institutions de l'enseignement supérieur et de la recherche ${ }^{4}$ [voir encadré, p. XX].

Une grande proximité entre le champ de l'histoire académique et le champ militant féministe caractérise ces premiers temps de l'institutionnalisation de l'histoire des femmes :

1. Natalie Zemon Davis, "Women and the world of the 'annales", History Workshop Journal, 33, 1992, p. 121137 et en particulier p. 121.

2. Bonnie G. Smith, The Gender of History. Men, Women, and Historical Practice, Cambridge/Londres, Harvard University Press, 1998.

3. Alice Primi, «Savoirs des marges, savoirs concurrents. Écriture et relecture de l'histoire par les "féministes" du Second Empire », in Jacqueline Carroy, Nicole Edelman, Annick Ohayon et Nathalie Richard (dir.), Les Femmes dans les sciences de l'homme (XIX ${ }^{e}-X X^{e}$ siècles). Inspiratrices, collaboratrices ou créatrices ?, Paris, Seli Arslan, 2005, p. 272-286.

4. Françoise Thébaud, Écrire l'histoire des femmes et du genre, Lyon, ENS Éd., 2007 ; Françoise Thébaud, «L'aventure intellectuelle de l'histoire des femmes en France », in Nicole Racine et Michel Trebitsch (dir.), Intellectuelles. Du genre en histoire des intellectuels, Bruxelles, Complexe, 2004, p. 311-338. 
les actrices, la démarche revendiquée et la correspondance des agendas (militant et de recherche) témoignent de la porosité des frontières entre espace associatif autonome et institutions, pendant la décennie 1970. Cette première phase de l'histoire des femmes, qualifiée par Michelle Perrot «d'exploration mémoriale et de quête identitaire ${ }^{5}$, se caractérise par une imbrication de la démarche historienne - qui consiste à produire des connaissances scientifiques sur le passé des femmes selon les règles de la discipline - et de la démarche mémorielle - que l'on pourrait définir ici comme la production d'un récit du passé à des fins identitaires et politiques ${ }^{6}$. Mais à partir du début des années 1980, l'histoire des femmes prend progressivement ses distances par rapport au militantisme et, parce qu'elle vise une légitimité institutionnelle, s'engage « dans un mouvement d'objectivation qui garantisse sa scientificité au même titre que toute autre spécialisation disciplinaire ${ }^{7}$. Cette stratégie d'intégration au champ académique se traduit, dans les recherches, par un évitement de l'objet mémoire, sorte de prise de distance des historiennes des femmes à l'égard du politique. Les années 1990 marquent cependant les limites de ce processus d'externalisation de la cause des femmes : face aux résistances des institutions de recherche et d'enseignement supérieur et sous l'effet du développement de l'histoire des féminismes, les historiennes des femmes renouent avec des normes et des pratiques militantes et s'investissent plus directement dans les usages politiques du passé, en collaboration avec les autres pôles de l'espace de la cause des femmes.

À partir de l'étude de l'historiographie féministe, de récits de vie et d'écrits autobiographiques d'historiennes des femmes, et à partir de l'analyse des archives personnelles de Christine $\mathrm{Bard}^{8}$, cet article propose de considérer le rapport des historiennes des femmes à la mémoire afin d'éclairer la trajectoire d'institutionnalisation de l'histoire des femmes depuis le début des années 1970. Ainsi, en analysant à la fois comment les historiennes se saisissent de la mémoire comme objet d'étude et comment elles s'engagent

5. Michelle Perrot, «Les premières expériences », Les cahiers du CEDREF, 10, 2001, p. 13-22 [en ligne].

6. Sur les processus de construction des mémoires collectives dans des groupes militants voir : Marie-Claire Lavabre, Le Fil rouge. Sociologie de la mémoire communiste, Paris, Presses de Sciences po, 1994 ; Richard A. Couto, "Narrative, free space, and political leadership in social movements", The Journal of Politics, 55(1), 1993, p. 57-79 ; Charles M. Payne, I’ve Got the Light of Freedom. The Organizing Tradition and the Mississippi Freedom Struggle, Berkeley, University of California Press, 1995.

7. F. Thébaud, Écrire l'histoire des femmes et du genre, op. cit., p. 174.

8. Christine Bard est historienne, spécialiste notamment du féminisme, professeure à l'Université d'Angers. Elle est engagée dans de nombreuses initiatives à vocation mémorielle. Elle a fondé le Centre des archives du féminisme, qui se trouve à la bibliothèque universitaire de Belle Beille à Angers, et préside depuis sa création en 2000, 1'association Archives du féminisme. Elle est également la co-fondatrice et la coordinatrice scientifique de Musea, le premier musée virtuel d'histoire des femmes et du genre, édité par l'Université d'Angers. 
dans des pratiques de construction du passé à des fins politiques, nous souhaitons mettre au jour l'évolution du rapport de l'histoire des femmes à la cause des femmes, selon les méandres de son institutionnalisation.

\section{De l’ « exploration mémoriale » au dessein scientifique, années 1970-1980}

Les premiers travaux en histoire des femmes sont fortement influencés par la sphère militante tant du point de vue de la perspective adoptée, des sujets d'études choisis, que des références intellectuelles mobilisées. Les historiennes des femmes assument d'ailleurs volontiers dans leurs écrits «une dimension engagée, justicière à l'égard des victimes des "silences de 1'histoire" » ${ }^{9}$. À partir du milieu des années 1980, elles se confrontent aux contradictions entre normes militantes et normes académiques et s'engagent dans un processus d'intégration institutionnelle. Leur volonté de se distancier du militantisme féministe, et du politique en général, les conduit paradoxalement à négliger la mémoire comme objet d'études, au moment même où ce dernier est perçu par leurs collègues historiens comme un instrument de renouvellement de la discipline.

\section{L'histoire des femmes comme projet mémoriel}

L'institutionnalisation de l'histoire des femmes est le fruit de la rencontre entre des actrices qui œuvrent dans le champ académique et d'autres dans le champ militant : d'un côté, la génération des «bâtisseuses », composée de femmes (telles Yvonne Knibiehler, Michelle Perrot, Françoise Basch, Madeleine Rebérioux, Rolande Trempé) déjà en poste à l'université dans les années 1970, spécialistes de l'histoire sociale et ouvrière, qui s'appuient sur leur ancrage institutionnel pour faire exister l'histoire des femmes dans le champ intellectuel ; de l'autre côté, la génération des «étudiantes», composée «de femmes (et d'hommes) d'origines et d'âges très divers, impliquées le plus souvent dans le Mouvement de libération des femmes (MLF) ou les mouvements homosexuels $»^{10}$, qui concilient engagement personnel et projet professionnel en donnant à leurs sujets d'études une orientation féministe. Cette

9. Christine Bard, «Les usages politiques de l'histoire des femmes », in Claire Andrieu, Marie-Claire Lavabre et Danielle Tartakowsky (dir.), Politiques du passé. Usages politiques du passé dans la France contemporaine, Aix-en-Provence, Presses universitaires de Provence, 2006, p. 71-82.

10. Sylvie Chaperon, «Du féminisme à la sexologie : un parcours en Histoire », Genre, sexualité \& société, 4, 2010 (http://journals.openedition.org/gss/1672). 
génération, à laquelle appartiennent, entre autres, Marie-France Brive, Marie-Jo Bonnet, Geneviève Fraisse, Françoise Picq ou Françoise Thébaud, constitue le socle d'une demande de création de nouveaux cours ${ }^{11}$ et fait usage des savoirs produits dans le mouvement social pour stimuler les réflexions académiques. Michelle Perrot parle d'un « désir militant, souvent extérieur à l'université, mais qui prenait appui sur elle $»^{12}$. Les femmes de la génération des « étudiantes », qui font le choix de l'histoire, ont pour la plupart une expérience militante dans les mouvements de gauche et/ou dans le MLF et affirment leur volonté d'accéder aux institutions sans toutefois s'y soumettre. Françoise Picq ${ }^{13}$, par exemple, explique qu'avec ses camarades, elles entendent faire du Groupe d'études féministes (GEF) de l'Université Paris 7 «le cheval de Troyes du MLF à l'université ${ }^{14}$. Soucieuses de rester en accord avec les normes en vigueur dans le MLF, l'objectif est de réussir à imposer à l'institution certains questionnements et modes de fonctionnement du mouvement social (horizontalité, non mixité, valorisation des savoirs fondés sur l'expérience).

Le refus des hiérarchies traditionnelles et l'ouverture à la parole des «étudiantes » permettent la diffusion d'interrogations et de théories politiques importées du mouvement social et absentes de l'histoire «classique». Le clivage entre savoirs savants et savoirs militants est contesté, et la distinction entre questionnements historiques et interrogations mémorielles est dès lors inopérante, comme l'explique Françoise Picq ci-dessous : «L'histoire du féminisme, telle que nous l'avons entreprise n'était guère conforme aux canons universitaires. Nous n'avions pas nécessairement de formation historique et refusions d'être enfermées dans un champ disciplinaire. Notre objectif était de lever la censure sur le féminisme passé, de mettre à l'honneur celles que nous appelions les "grands-mères" avec lesquelles nous avions un rapport pas du tout distancié. Chacune d'entre nous avait sa "chouchoute" : Hélène Brion, Madeleine Pelletier, Hubertine Auclert, Madeleine Vernet ou les Saint-Simoniennes. Nous ne nous intéressions qu'à celles chez lesquelles nous trouvions un écho à nos propres préoccupations. Nous découvrions avec enthousiasme les bribes de

11. Soulignons que le développement de l'histoire des femmes à l'université est favorisé par deux phénomènes concomitants : l'existence d'une forte demande sociale émanant du mouvement des femmes et une progression importante du nombre d'étudiantes à l'université. D'après le site internet de l'Observatoire des inégalités, elles passent de 42,8\% des effectifs globaux en $1960-1961$ à $49,75 \%$ en $1980-1981$ (http://www.inegalites.fr/spip.php?article1096\&id_mot=124).

12. M. Perrot, «Les premières expériences », art. cit.

13. Pour des éléments sur sa trajectoire, voir Marion Charpenel, «Françoise Picq », in Christine Bard (dir.), Dictionnaire des féministes, France XVIII ${ }^{e}$-XXI ${ }^{e}$ siècle, Paris, PUF, 2017, p. 1131-1134.

14. Françoise Picq, «Du mouvement des femmes aux études féministes », Les cahiers du CEDREF, 10, 2001, p. 23-31. 
leurs vies et nous les partagions. Ce dont nous n'avions pas conscience, c'est qu'à travers l'histoire du féminisme, c'est la nôtre qui nous inquiétait. "Allons-nous re-disparaître sans avoir fait dérailler l'histoire et le chef de gare ?" ${ }^{15}$

Outre ce constat spécifique à l'histoire du féminisme selon lequel «le besoin d'identification des historiennes et de leurs lectrices éclair[e] le choix des périodes, des personnalités et des mouvements les plus étudiés ${ }^{16}$, de manière plus générale, l'actualité de la lutte influence les travaux et des sujets abondamment discutés dans les groupes de parole du MLF, comme la sexualité, sont constitués en objets d'études.

«[Mes travaux] partent des prises de parole féminine sur l'accouchement et des critiques contre le pouvoir médical ou le fonctionnement des maternités : "Donner la vie. Histoire de la maternité en France entre les deux guerres" ${ }^{17}$ voulait montrer sur un temps court l'historicité de la maternité, “tout comme le statut et le sentiment de l'enfant ne sont pas des données immuables mais historiquement déterminées"18. »

Le renouvellement des thématiques, comme le corps, la maladie, l'enfermement doit beaucoup à l'influence d'auteurs comme Michel Foucault, souvent découverts dans les milieux militants, mobilisés plutôt par d'autres disciplines, mais importés en histoire via les recherches sur les femmes.

On observe ainsi une « continuité de pratiques ou d'horizon intellectuel et politique ${ }^{19}$ entre l'histoire des femmes et le MLF, qui donne lieu à la coexistence de deux histoires des femmes, l'une plutôt misérabiliste et l'autre réhabilitatrice, toutes deux offrant aux militantes des ressources pour la lutte. Tout d'abord, il y a « la femme victime, voire opprimée par une domination inhérente aux rapports de sexe $»^{20}$ - Sheila Ryan Johansson identifie également cette figure dans la littérature étasunienne et la nomme « Woman-the-passive-victim ${ }^{21}$. Elle

\section{Ibid.}

16. Christine Bard, «Introduction», in Christine Bard, Annie Metz, et Valérie Neveu (dir.), Guide des sources de l'histoire du féminisme, Rennes, PUR, 2006, p. 10.

17. Titre de la thèse soutenue par Françoise Thébaud dans le cadre de son doctorat de $3^{\mathrm{e}}$ cycle, sous la direction de Michelle Perrot.

18. F. Thébaud, Écrire l'histoire des femmes et du genre, op. cit., p. 81.

19. Claude Zaidman, «Enseigner le féminisme ? Transmission instituée et rapports de générations », in Groupe d'études féministes de l'Université Paris 7 (dir.), Crises de la société, féminisme et changement: Actes du colloque, Paris, Sorbonne, 22-23 avril 1988, organisé par le GEF, Paris, Tierce, 1991, p. 245-256.

20. Michelle Perrot (dir.), «La bibliothèque, mère de l'histoire des femmes », Revue de la Bibliothèque nationale de France, 17, 2004, p. 19-24.

21. Sheila Ryan Johansson, "'Herstory" as history: a new field or another fad", in Berenice A. Carroll (dir.), Liberating Women's History. Theorical and Critical Essays, Urbana/Chicago/Londres, University of Illinois Press, 1976, p. 400-430. 
s'incarne par exemple dans les travaux sur les «bonnes $»^{22}$ ou les prostituées ${ }^{23}$. En mettant l'accent sur les oppressions dont sont victimes les femmes, ces recherches apportent la preuve de la permanence de l'oppression, et permettent aux militantes de justifier leurs luttes. La seconde figure qui émerge de ces recherches des premiers temps est celle de la femme porteuse d'une culture féminine spécifique, dont on fait l'histoire « des gestes, de l'oralité et des objets, fondée sur la reproduction, dans un temps impalpable et un espace restreint $»^{24}$. Les travaux sur les «métiers de femmes » (ouvrières, sages-femmes, infirmières, etc. $)^{25}$, mais aussi ceux sur les «femmes au foyer $»^{26} s^{\prime}$ inscrivent dans cette perspective. On cherche à comprendre comment au quotidien, dans la maison, au travail ou dans des lieux de sociabilité typiquement féminins (comme le lavoir, le marché, le four, etc.), les femmes ont développé des «façons de dire, façons de faire $»^{27}$. Cette histoire ouvre la voie à un questionnement sur le pouvoir (parfois caché) des femmes et sur leurs modalités d'intervention dans la vie publique. L'ouvrage collectif L'Histoire sans qualités, paru en 1979, annonce ce changement et met l'accent sur les marges de manœuvre que se ménagent les femmes pour s'extraire de leur condition, plutôt que sur les pesanteurs qui les assignent à tel ou tel statut.

Sans se départir d'une méthode scientifique, l'histoire des femmes à ses débuts se pose ainsi comme un réservoir de modèles, d'exemples, de ressources identitaires pour les militantes de la cause des femmes.

\section{Les contradictions de l'intégration}

Très rapidement, la proximité entre champ associatif autonome et champ académique révèle des contradictions susceptibles de menacer l'unité d'un espace de la cause des femmes naissant. Membres du GEF et d'un courant du MLF appelé «groupe du jeudi » ${ }^{28}$, Françoise

22. Anne Martin-Fugier, La Place des bonnes. La domesticité féminine à Paris en 1900, Paris, Grasset \& Fasquelle, 1979.

23. Alain Corbin, Les Filles de noce. Misère sexuelle et prostitution, XIX et $X X^{e}$ siècles, Paris, AubierMontaigne, 1978 ; Jacques Termeau, Maisons closes de province : l'amour vénal au temps du réglementarisme à partir d'une étude du Maine-Anjou, Le Mans, Cénomane, 1986.

24. M. Perrot, « La bibliothèque, mère de l'histoire des femmes », art. cit., p. 19.

25. Katherine Blunden, Le Travail et la vertu : femmes au foyer, une mystification de la révolution industrielle, Paris, Payot, 1982.

26. Anne Martin-Fugier, La Bourgeoise : femme au temps de Paul Bourget, Paris, Grasset \& Fasquelle, 1983.

27. Yvonne Verdier, Façons de dire, façons de faire : la laveuse, la couturière, la cuisinière, Paris, Gallimard, 1979.

28. Pour plus d'éléments sur ce groupe, voir Jane Jenson, «Le féminisme en France depuis mai 68 », Vingtième Siècle. Revue d'histoire, 24, 1989, p. 55-67. 
Picq et Liliane Kandel $^{29}$ se souviennent des résistances de certaines militantes du MLF vis-àvis de l'institutionnalisation.

«Et je me souviens encore du jour où des amies du mouvement sont venues au Groupe d'études féministes (GEF) à Paris 7 pour nous reprocher de faire de l'histoire plutôt que des avortements... Ou du groupe auto-intitulé "mongoliennes", opposé aux "intellos" du mouvement, contre qui nous soutenions mordicus que si l'Histoire ne marchait pas sur la tête, elle ne pensait pas non plus avec son utérus, et que nous étions entêtées et non étêtées ${ }^{30}$. »

Les arguments avancés par les plus hostiles au déploiement de la contestation féministe dans l'université sont d'une triple nature : elles contestent le fait que des femmes s'engagent dans des institutions qui sont un «lieu de compétition professionnelle, apanage des hommes $»^{31}$. Elles regrettent aussi le fait qu'en s'engageant dans le champ académique, les chercheuses tirent un profit personnel de la lutte des femmes et s'en servent pour se distinguer de «toutes les femmes $»^{32}:$ «Vendre la lutte des femmes à l'université » est dénoncé comme un acte de traitrise. Enfin, elles critiquent le caractère androcentrique du discours scientifique et doutent de sa capacité à théoriser une oppression que seules les opprimées peuvent penser.

Ces critiques et l'essoufflement de la sphère militante après les mobilisations pour la reconduction de la loi sur l'interruption volontaire de grossesse en $1979^{33}$ incitent les historiennes des femmes à se «détourner de la centralité de la cause des femmes, de ses enjeux et intérêts spécifiques $»^{34}$, et à investir davantage le champ académique, plus professionnalisé et plus gratifiant que l'espace associatif autonome des années 1980. La question de la mixité et l'usage de l'expression «histoire féministe» mettent au jour l'existence de contradictions entre normes académiques et pratiques militantes et font l'objet de compromis de la part des féministes insérées dans les institutions. Alors que les «étudiantes », influencées par leurs expériences au sein du MLF, voient dans les séminaires

29. Liliane Kandel est sociologue, membre du Laboratoire de psychologie sociale (CNRS) à cette époque, elle est également militante au MLF à partir de décembre 1970. Voir Isabelle Lacoue-Labarthe, «Kandel Liliane », in C. Bard (dir.), Dictionnaire des féministes..., op. cit., p.792-795.

30. Margaret Maruani et Nicole Mosconi, «Liliane Kandel, Génération MLF », Travail, genre et sociétés, 24, 2010, p. 5-24.

31. Rose-Marie Lagrave, «Recherches féministes ou recherches sur les femmes? », Actes de la recherche en sciences sociales, 83, 1990, p. 27-39 et en particulier p. 28.

32. F. Picq, «Du mouvement des femmes aux études féministes », art. cit.

33. Bibia Pavard, Si je veux, quand je veux. Contraception et avortement dans la société française (1956-1979), Rennes, PUR, 2012.

34. Laure Bereni, «Penser la transversalité des mobilisations féministes : l'espace de la cause des femmes », in Christine Bard (dir.), Les Féministes de la deuxième vague, Rennes, PUR, 2012, p. 27-41 et en particulier p. 38. 
des lieux d'émancipation, mais à la condition d'en exclure les hommes en position d'y monopoliser la parole, les impératifs de neutralité et d'universalisme de l'enseignement, dominants à l'université, empêchent le transfert direct de la norme militante de non-mixité et imposent des compromis: la faible importance numérique des hommes permet, de fait, l'existence d'espaces non mixtes, mais officiellement les cours restent ouverts aux hommes conformément aux exigences académiques ${ }^{35}$. De la même façon, le soupçon de science militante porté par leurs collègues conduit les historiennes françaises, contrairement aux Américaines, à rejeter l'expression « histoire féministe » (feminist history en anglais) pour lui préférer une dénomination plus euphémisée, « histoire des femmes $»^{36}$.

De manière générale, les opportunités politiques ouvertes par la victoire de la gauche à l'élection présidentielle de 1981 conduisent l'ensemble des féministes à repenser leur rapport aux institutions. Après des années 1970, globalement dominées par la radicalité, il s'agit de choisir entre deux orientations : d'un côté, le maintien d'une position contestataire - qui implique un certain renoncement face aux opportunités suscitées par le changement de majorité ; de l'autre, l'adaptation aux contraintes imposées par la collaboration avec les institutions pour réaliser ce qui, jusqu'alors, appartenait au registre de l'idéal ou de l'utopie. Toutes les féministes, selon leur trajectoire et leur ancrage social, n'ont pas la même position à l'égard de ce dilemme, mais dans l'ensemble les historiennes des femmes, à la suite des sociologues et des anthropologues, décident de prendre le «tournant institutionnel » et de profiter de ce nouveau contexte, propice à la reconnaissance de leur champ d'études par le pouvoir scientifique. En effet, la nomination au poste de directeur du Département des sciences de l'homme et de la société du CNRS de Maurice Godelier, anthropologue spécialisé dans l'analyse des rapports de parenté, marque l'ouverture relative de cette institution aux études féministes ${ }^{37}$. Les années 1982-1983 accélèrent ainsi le processus d'institutionnalisation : un Colloque national des études féministes destiné à dresser un état des lieux des recherches féministes en France ${ }^{38}$ se tient à Toulouse ; une Action thématique programmée est mise en place sur cinq ans, dotée d'un conseil scientifique composé

35. Sur l'ouverture officielle aux hommes sans qu'aucune action ne soit mise en œuvre pour les attirer réellement, voir Alban Jacquemart, «Les hommes dans les mouvements féministes français (1870-2010). Sociologie d'un engagement improbable », thèse de sociologie, EHESS, 2011, p. 184.

36. F. Thébaud, Écrire l'histoire des femmes et du genre, op. cit., p. 52.

37. R.-M. Lagrave, art. cit., p. 30.

38. Liliane Kandel, «Un tournant institutionnel : le colloque de Toulouse », Les cahiers du CEDREF, 10, 2001, p. 81-101. Des financements sont également obtenus pour envoyer une importante délégation française au colloque international de Montréal sur « la recherche et l'enseignement relatifs à la femme » en août 1982. 
uniquement de chercheuses féministes ${ }^{39}$. Au sein des recherches féministes, coexistent plusieurs positions analysées par Rose-Marie Lagrave ${ }^{40}$. Contrairement à la psychologie et à la sociologie qui adoptent majoritairement des positions «militante » ou «médiane », l'histoire se situe principalement du côté de la recherche « orthodoxe ». C'est, par exemple, le cas $\mathrm{du}$ «groupe le plus dynamique et le plus productif en histoire des femmes ${ }^{41}$, le Séminaire d'histoire des femmes de l'EHESS, formé autour d'Arlette Farge, Christiane Klapisch-Zuber, Cécile Dauphin, Pierrette Pezerat, Michelle Perrot, Geneviève Fraisse, Danièle Voldman, Yannick Ripa et quelques autres, séminaire qui se donne pour ambition et pour stratégie de transformer la discipline historique en pratiquant une forme d'entrisme: «L'histoire est une discipline très masculine et l'École en est un des sanctuaires. Nous éprouvions le besoin d'y pénétrer et de nous y affirmer, notamment par rapport aux Annales ${ }^{42}$. 》

Comme le dit Michelle Perrot, les historiennes des femmes font le choix de «la pénétration plutôt que de la sécession ». Stratégie qui donne lieu à quelques victoires : ce groupe parvient ainsi à opérer une percée dans la prestigieuse revue des Annales, longtemps hermétique aux productions de l'histoire des femmes, avec notamment la publication d'un article collectif en 1986, intitulé «Culture et pouvoir des femmes : essai d'historiographie » et, plus tard, en 1993, un dossier sur « Histoire des femmes, histoire sociale ».

\section{L'évitement de l'objet mémoire}

Ainsi, dès la fin des années 1970, un fossé se creuse entre les historiennes des femmes et la sphère militante, qui se traduit par une distance entre histoire et mémoire. Les chercheuses privilégient une histoire savante dégagée des motivations mémorielles des premiers temps ; les militantes commencent à construire le récit des années 1970 élevées au rang d'âge d'or des féminismes; et des féministes investies dans des instances étatiques se saisissent du passé à des fins politiques, sans lien manifeste avec l'actualité de la recherche historique ${ }^{43}$. Un autre

39. Françoise Barret-Ducrocq, «L'invention d'un nouveau champ de recherche », Les cahiers du CEDREF, 10, 2001, p. 33-36.

40. R.-M. Lagrave, art. cit.

41. F. Thébaud, Écrire l'histoire des femmes et du genre, op. cit.

42. M. Perrot, «Les premières expériences », art. cit.

43. L'histoire des femmes, qui brillait jusqu'alors par son absence dans le récit national, accède progressivement à une reconnaissance officielle par la voix d'Yvette Roudy, ministre des Droits de la femme, et de ses usages politiques du passé. Cette dernière, dans ses rappels du passé, met surtout l'accent sur la valorisation de femmes exceptionnelles par leurs parcours personnels ou leurs engagements féministes. Cette politique mémorielle, qui a 
constat confirme ce processus de séparation entre histoire et mémoire au sein de l'espace de la cause des femmes dans les années 1980 : les historiennes des femmes montrent peu d'intérêt pour l'objet mémoire, qui pourtant, au même moment, conquiert une place centrale dans la discipline.

L'historiographie française des années 1980 est en effet marquée par ce que Pierre Nora appelle le «moment-mémoire ». Ce moment débute plus exactement à la fin des années 1970 avec deux articles de Pierre Nora, en 1978 et 1979, dans lesquels il donne une définition de la mémoire collective et souligne d'emblée «l'utilisation stratégique » que les historiens peuvent faire de cette notion pour renouveler la discipline et susciter une réflexion sur les conflits d'interprétations et la relativité des connaissances en cours ${ }^{44}$. Avec la parution en 1983 de l'ouvrage de Philippe Joutard, Ces voix qui nous viennent du passé, la publication en 1984 du premier tome des Lieux de mémoire, dirigé par Pierre Nora, celle en 1987 du Syndrome de Vichy de Henry Rousso, et celle en 1989 de La Vendée de la mémoire (18001980) de Jean-Clément Martin, la mémoire devient alors un véritable objet d'histoire. Malgré ce contexte, les historiennes des femmes, pourtant parmi les premières à investir le champ de l'histoire orale, ne participent pas à cette inflation des recherches et des publications sur la mémoire. Le séminaire d'histoire orale, animé par Danièle Voldman et Sylvie Schweitzer depuis avril 1982, à l'Institut d'histoire du temps présent (IHTP), par exemple, s'arrête en juin 1983, c'est-à-dire au moment même de la sortie de l'ouvrage de Philippe Joutard, et peu avant celle des Lieux de mémoire.

Cette ignorance mutuelle se manifeste, tout d'abord, par le peu de place accordée aux femmes dans la grande entreprise des Lieux de mémoire, dont les derniers tomes paraissent pourtant l'année de la publication du dernier volume de la série Histoire des femmes en Occident. Michelle Zancarini-Fournel souligne avec ironie le paradoxe présent dans la contribution de Michelle Perrot à l'entreprise des Lieux de mémoire censée exposer des

vocation à ancrer la mémoire féministe dans une mémoire de gauche, montre l'écart qui existe entre ces actions centrées sur la valorisation d'individualités et l'historiographie savante de l'époque qui met l'accent sur les forces profondes de la société, les mentalités, les conduites ordinaires, et est « hostile à la personnalisation des luttes collectives ». Voir Christine Bard, «Les usages politiques de l'histoire des femmes », Bulletin Archives du féminisme, 2003 (http://www.archivesdufeminisme.fr/article.php3?id_article=79) ; Marion Charpenel, «"Le privé est politique !" : sociologie des mémoires féministes en France », thèse de doctorat en science politique, Paris, Institut d'études politiques, 2014.

44. Pierre Nora, « Mémoire collective », in Jacques Le Goff, Roger Chartier et Jacques Revel (dir.), La Nouvelle Histoire, Paris, Retz-CEPL, 1978, p. 398-401; Pierre Nora, "Quatre coins de la mémoire », Histoire, 2, 1979, p. 9-32 ; Marie-Claire Lavabre, «Usages et mésusages de la notion de mémoire », Critique internationale, 7, 2000, p. 48-57 et en particulier p. 49. 
biographies ouvrières, mais rabattues sur celles d'ouvriers ${ }^{45}$. Plus généralement, pendant les années 1980, la mémoire des femmes apparaît comme la grande absente des recherches historiques sur la mémoire. Seule l'historienne et militante féministe Claire Auzias, qui soutient en 1980 une thèse sur la question de la mémoire dans les milieux libertaires lyonnais, met en évidence le rôle des femmes dans la construction et l'entretien du souvenir de ces mobilisations ${ }^{46}$.

Réciproquement, et hormis quelques rares publications, l'histoire des femmes ignore l'objet mémoire. Sur l'ensemble de la décennie 1980, seulement deux publications sont spécifiquement consacrées à la question de l'existence d'une mémoire de femmes ou féministe. Elles sont coordonnées par Danièle Voldman et Sylvie Schweitzer, membres depuis 1981 de l'IHTP, laboratoire auquel appartiennent des chercheurs comme Henry Rousso, Denis Peschanski, Jean-Pierre Rioux ou encore Michael Pollak, qui placent l'objet mémoire au cœur de leurs interrogations scientifiques. Ce contexte professionnel, couplé à l'intérêt de ces deux chercheuses pour l'histoire orale, les incitent à publier sur cette question. Tout d'abord, en 1982, le Bulletin de l'IHTP retranscrit une table ronde tenue le 16 octobre 1981 sur « Histoire orale et histoire des femmes ». Une partie de la table ronde est organisée autour de la question «Quelle mémoire féminine?», au cours de laquelle une soixantaine de chercheurs essayent de répondre à la question suivante : «Quelle mémoire les femmes gardent-elles des faits et des grands événements de leur passé ? ${ }^{47}$. La conclusion qui semble se dégager, reprise par Sylvie Schweitzer et Danièle Voldman dans leur chapitre de l'ouvrage de Michelle Perrot, Une histoire des femmes est-elle possible ?, avance l'idée selon laquelle il faut «abandonner l'hypothèse d'une mémoire spécifique aux femmes ${ }^{48}$ et considérer que la mémoire est façonnée selon les itinéraires individuels et les rôles sociaux plutôt que selon le $\operatorname{sexe}^{49}$. Pour Michelle Zancarini-Fournel, les historiennes des femmes sont réticentes à isoler des sujets-femmes qui seraient porteuses d'une mémoire spécifique parce qu'elles refusent de construire une histoire des femmes séparée de l'histoire globale. On retrouve cette même

45. Michelle Zancarini-Fournel, «Mémoires et histoire des femmes : histoire d'un évitement des sources orales à l'avènement du Je », communication au colloque «Histoire orale, histoire des femmes, mémoire des femmes : pratiques et débats d'hier et d'aujourd'hui », organisé par Françoise Thébaud, en 2006.

46. Claire Auzias, «Mémoires libertaires : Lyon 1919-1939 », thèse de doctorat en histoire contemporaine, Lyon, Université Lumière Lyon 2, 1980.

47. IHTP, «La vie de l'Institut d'histoire du temps présent », Bulletin de l'Institut d'histoire du temps présent, 6 , 1981, p. 3-13.

48. Sylvie Schweitzer et Danièle Voldman, «Les sources orales pour l'histoire des femmes », in Michelle Perrot (dir.), Une histoire des femmes est-elle possible ?, Marseille, Rivages, 1984, p. 63-64.

49. Dont on sait pourtant qu'il constitue un rôle social. 
méfiance vis-à-vis de l'idée d'une mémoire «féminine » dans le numéro spécial de la revue Pénélope intitulé « Mémoires de femmes », paru en 1985.

«S'il n’y a plus, à notre sens, de spécificité du champ historique “femmes", il n’y a pas, non plus, de mémoire "féminine"; que serait celle - cela s'est dit - du privé et des chronologies familiales ${ }^{50}$. »

Ce cahier, coordonné par Danièle Voldman et Sylvie Schweitzer, composé de deux parties (l'une intitulée «Discipliner la mémoire », et l'autre « Mémoire de femmes»), témoigne dès l'introduction de l'embarras des spécialistes de l'histoire des femmes dès lors qu'il s'agit de parler de mémoire au milieu des années 1980: «Mémoire des femmes? Femmes et mémoire... Mémoires de femmes. Sur le titre de ce numéro, la rédaction de Pénélope, nous-mêmes et donc les auteurs, ont, un temps, hésité. S'agissait-il de cette mémoire appréhendée par les sources orales, ce biais privilégié pour écrire l'histoire des opprimés et des marginaux ? Et devait-on miser sur l'apitoiement suscité par tous ces exclus des bibliothèques ? S'agissait-il d'isoler une mémoire spécifique aux femmes ? Ou devait-on s'interroger sur la nécessité d'articuler mémoire et histoire ${ }^{51}$ ?»

La prise de distance de l'histoire des femmes à l'égard des motivations mémorielles des premiers temps est également perceptible dans ce numéro : «Nous avons pensé montrer que, tout autant que possible, une "histoire des femmes" est nécessaire, mais assortie de conditions : point trop d'attendrissement militant, guère de partage des champs entre les opprimés et les "autres" déjà choyés par les études des savants ${ }^{52}$. »

Ces historiennes sont donc partagées entre, d'un côté, leur volonté de se saisir de la mémoire comme un objet de recherche pour en comprendre le fonctionnement (en faisant notamment appel à des sociologues, des anthropologues et des philosophes pour compléter leur démarche), et de l'autre côté, leur souci de manifester leur attachement à une histoire savante qui relègue la mémoire au rang de question politique.

Ce malaise autour de l'objet mémoire illustre la façon dont l'institutionnalisation de l'histoire des femmes détourne provisoirement ses spécialistes des enjeux qui occupent les militantes de la cause des femmes (du pôle étatique et du pôle associatif). Il faut attendre le tournant des années 1990 - particulièrement les thèses sur les mouvements féministes de Laurence Klejman, Florence Rochefort, Christine Bard, et Sylvie Chaperon - pour voir de

50. Sylvie Van De Casteele-Schweitzer et Danièle Voldman, «Introduction », Pénélope, 12, 1985, p. 3-4.

51. Ibid.

52. Ibid. 
nouveau des historiennes des femmes et des féminismes poser la question de la mémoire. Cette période marque d'ailleurs chez les historiennes des femmes en général un regain d'intérêt (scientifique et militant) pour la mémoire.

\section{Les historiennes militantes de la mémoire, années 1990-2000}

Le processus d'externalisation de la cause des femmes dans l'enseignement et la recherche amorcé dans les années 1980 se heurte à des résistances : la question de l'objectivité est sans cesse soulevée à propos des travaux sur les femmes, et «la hiérarchie des territoires de l'historien avec au sommet, le politique et le pouvoir ${ }^{53}$ perdure malgré les évolutions que connaît la discipline. La fin des années 1980 et le début des années 1990 marquent donc les limites de l'intégration du féminisme dans le champ académique. Contre le désintérêt de l'historiographie «générale » vis-à-vis des résultats de l'histoire des femmes et devant le faible nombre de postes finalement obtenus, les historiennes des femmes renouent alors avec une posture militante. En travaillant à la conservation et à la valorisation des traces et en s'engageant dans des formes variées de lutte pour une meilleure visibilité du passé des femmes dans la société, elles endossent le rôle d' « entrepreneuses de mémoire ${ }^{54}$.

\section{Le retour de la position militante dans l'institution}

À l'occasion d'un colloque organisé par le GEF en 1988, Françoise Picq s'interroge sur la pertinence de la voie prise par les historiennes des femmes dans les années 1980 : «Avonsnous donc, pour mieux écrire notre histoire en "universitaires", cessé d'être des "activistes" ? Avons-nous sacrifié nos objectifs politiques sur l'autel de la reconnaissance institutionnelle et opéré un excessif "détachement" ?5 »

53. Brigitte Studer et Françoise Thébaud, «Entre histoire et mémoire », in Eliane Gubin et al. (dir.), Le Siècle des féminismes, Ivry-sur-Seine, Éd. de l'Atelier, 2004, p. 27-45.

54. Michael Pollak définit ainsi des «acteurs professionnalisés » qui effectuent un «travail d'encadrement de la mémoire ». Il s'inscrit dans la continuité de «l'analyse que fait Howard S. Becker des "entrepreneurs de morale" » et parle, "par analogie, d'entrepreneurs de mémoire, qui se composent de deux catégories : ceux qui créent les références communes et ceux qui veillent à leur respect ». Voir Michael Pollak, Une identité blessée. Études de sociologie et d'histoire, Paris, Métailié, 1993, p. 30.

55. Françoise Picq, «Si c'était à refaire? », in Groupe d'études féministes de l'Université Paris 7 (dir.), Crises de la société, féminisme et changement, op. cit., p. 260. 
Cette interrogation est révélatrice des questions qui se posent aux historiennes des femmes au début des années 1990. Cette période est marquée par la parution des cinq volumes de l'Histoire des femmes en Occident codirigée par Georges Duby et Michelle Perrot. Mais la réception médiatique et populaire de l'œuvre contraste avec sa réception scientifique.

«En ce qui concerne les lecteurs, particulièrement les collègues masculins du monde universitaire, il nous semble que, sous l'éloge, se fige une grande indifférence (avec des exceptions notables bien sûr); ce qui veut dire en somme, que cette histoire-là risque d'affecter bien peu la manière habituelle de faire de l'histoire. Une once d'admiration, un silence sceptique, et le tour est joué ${ }^{56}$. »

Si Michelle Perrot justifiait sa participation à l'Histoire des femmes en Occident par son ambition de sortir l'histoire des femmes du «ghetto toujours possible $»^{57}$, elle dresse le constat que cette entreprise colossale ne suffise pas à convertir ses collègues historien-ne-s à une plus grande prise en compte des femmes dans leurs propres travaux, ni ne les incite à mieux valoriser les recherches issues de ce nouveau champ d'études.

La désillusion est d'abord épistémologique. Les historiennes des femmes prennent conscience des multiples contraintes les amenant à se «couler dans le moule » de la discipline. À titre d'exemple, le découpage chronologique choisi pour structurer l'Histoire des femmes en Occident correspond aux périodes classiques de l'enseignement de l'histoire en France et ne parvient pas à imposer « une temporalité qui pourrait, qui devrait être autre "que celle empruntée à l'historiographie traditionnelle" $»^{58}$. De plus, le fait que les femmes aient laissé peu de traces dans les archives en raison de leur exclusion de la sphère publique, restreint les possibilités d'accès à des sources nouvelles et oblige les historiennes des femmes à relire «en creux» les sources existantes et à «faire surgir, par le regard qu'[elles] leur porte[nt], les femmes ou la division sexuelle ${ }^{59}$. Pour Geneviève Fraisse, elles ne peuvent pas « faire du neuf, mais plutôt "du neuf avec de l'ancien" » ${ }^{60}$. Outre cette question des sources, le fait d'entrer dans l'histoire des femmes par l'angle des représentations a également restreint le

56. Arlette Farge et Michelle Perrot (dir.), «Débat», in Georges Duby et Michelle Perrot (dir.), Femmes et histoire, contributions présentées à un colloque avec les directrices de «Histoire des femmes en Occident », Paris, Plon, 1993, p. 72.

57. Michelle Perrot, « Où en est en France l'histoire des femmes?», French Politics and Society, 12(1), 1994, p. 39-57.

58. Roger Chartier, «L'Histoire des femmes, $X V I^{\mathrm{e}}-\mathrm{XVIII}{ }^{\mathrm{e}}$ siècle. Différences entre les sexes et violence symbolique », in G. Duby et M. Perrot (dir.), Femmes et histoire..., op. cit., p. 45.

59. F. Thébaud, Écrire l'histoire des femmes et du genre, op. cit., p. 71.

60. Geneviève Fraisse, Les Femmes et leur histoire, Paris, Gallimard, 2010 [1998], p. 31. 
champ des possibles: parce qu'«elle s'est écrite à partir des discours, plus ou moins normatifs, qui parlent [des femmes], et des images qui les peignent $\gg^{61}$, 1'histoire des femmes est restée dépendante du regard masculin. Claude Mossé décrit le cercle vicieux dans lequel sont prises les historiennes des femmes : la pauvreté des sources les contraint à travailler à partir de textes écrits par les hommes sur les femmes, et la nécessité de déconstruire ce regard justifie la place privilégiée accordée aux représentations. Claude Mossé en appelle donc à la réintroduction des « réalités concrètes dans l'analyse ${ }^{62}$.

Outre cette prise de conscience du biais normatif imposé par les sources, le bilan que les historiennes des femmes font alors de leur insertion institutionnelle se révèle en demiteinte également. Devant l'échec de la stratégie d'intégration, elles s'engagent dans des formes plus militantes de lutte pour une meilleure place dans la discipline. Elles n'hésitent pas à mettre les tenants de la discipline face à leurs insuffisances, comme l'atteste l'intitulé du colloque «Une histoire sans les femmes est-elle possible ? » organisé à Rouen en 1997. Cette nouvelle posture revendicative est incarnée notamment par de jeunes historiennes du féminisme ${ }^{63}$ dont les objets d'études les ont familiarisées aux pratiques contestataires et aux activités collectives. Elles travaillent à la valorisation de l'histoire des femmes dans le champ académique en créant leurs propres structures (revues, associations et réseaux thématiques) ; et déploient ainsi concrètement la cause des femmes au sein des institutions de recherche et d'enseignement. L'une de ces revues, Clio. Histoire, femmes et sociétés, est fondée en 1995. Certes cette revue, animée par un comité de rédaction intergénérationnel ${ }^{64}$, affirme plus que son aînée Pénélope son insertion dans la discipline ${ }^{65}$; mais en assumant sa spécialisation, elle marque une nouvelle étape dans l'histoire des femmes.

« Nous n'étions pas retenues par la crainte, dépassée à nos yeux, de faire de l'histoire des femmes un "ghetto", crainte exprimée à plusieurs reprises par Pénélope et par de nombreuses historiennes dans les années 1970 et $1980^{66}$. »

61. M. Perrot, « La bibliothèque, mère de l'histoire des femmes », art. cit., p. 23.

62. Claude Mossé, «L'Antiquité. Lecture critique du tome 1 de L’Histoire des femmes », in G. Duby et M. Perrot (dir.), Femmes et histoire..., op. cit., p. 19.

63. La thèse de Laurence Klejman et Florence Rochefort est soutenue en octobre 1987, celle de Christine Bard en janvier 1994 et celle Sylvie Chaperon en décembre 1996.

64. Les membres du comité de rédaction au moment de sa création sont Christine Bard, Agnès Fine, Gabrielle Houbre, Christiane Klapisch-Zuber, Claudine Leduc, Catherine Marand-Fouquet, Florence Rochefort, Michelle Zancarini-Fournel, Françoise Thébaud.

65. Le choix du sous-titre, qui fait écho à celui de la célèbre revue des Annales et qui renonce à l'utilisation du terme « genre », témoigne de cette volonté d'être prises au sérieux.

66. Françoise Thébaud et Michelle Zancarini-Fournel, «CLIO, Histoire, Femmes et Sociétés : naissance et 
La peur d'être perçu comme un champ d'études autonome se délite et ce dernier peut ainsi se doter de formes associatives spécifiques assurant sa visibilité. En 2000, trois associations regroupant des historiennes des femmes se constituent : la Société internationale d'étude des femmes sous l'Ancien Régime (SIEFAR), l'association Archives du féminisme et mnémosyne (Association pour le développement de l'histoire des femmes et du genre). Les historiennes s'impliquent également dans la création et l'animation de réseaux ou associations pluridisciplinaires comme l'ANEF (Association nationale des études féministes), créée en 1991 ; le RING (Réseau interuniversitaire et interdisciplinaire sur le genre) créé en 2001 ; et EFiGiES (Association de jeunes chercheuses et chercheurs en études féministes, genre et sexualités) créée en 2003. Ces associations valorisent les travaux réalisés par des journées d'études ou en créant des prix récompensant thèses et mémoires en études féministes. Selon les moments, elles endossent également des revendications plus politiques : alors que les premières créations de postes fléchés en 1984 avaient été le résultat d'une initiative gouvernementale ${ }^{67}$, l'ANEF reprend l'initiative en 1991 et demande un rendez-vous à Lionel Jospin, alors ministre de l'Éducation nationale, pour argumenter en faveur de leur développement ${ }^{68}$. Si les historiennes soucieuses de ne pas «créer un ghetto intellectuel et institutionnel» et de ne pas «forger un champ d'études et des cursus tolérés mais dévalorisés ${ }^{69}$ considéraient avec méfiance les postes fléchés jusque dans les années 1990, elles rejoignent progressivement les sociologues et les psychologues dans leurs revendications.

\section{Des historiennes dans des actions mémorielles}

Moins soucieuses que dans les années 1980 de prendre leurs distances à l'égard de la sphère militante, les historiennes des femmes et du féminisme des années 1990 et 2000 renouent avec le politique et se laissent de nouveau saisir par des interrogations mémorielles venues de l'espace associatif autonome.

histoire d'une revue », CLIO, Histoire, femmes et sociétés, 16, 2002, p. 9-22.

67. Quatre postes d'études féministes avaient été concédés par le ministère de l'Éducation aux universités, dont un en histoire, celui de Marie-France Brive à Toulouse.

68. Françoise Picq, «Les études féministes en France : une institutionnalisation problématique », Labrys, études féministes/estudos feministas, $2005 \quad$ (http://francoisepicq.fr/site/wp-content/uploads/2016/06/Les$\% \mathrm{C} 3 \%$ A9tudes-f\%C3\%A9ministes-en-france.-Une-institutionnalisation-probl\%C3\%A9matique_2005_Labrys1.pdf).

69. F. Thébaud, Écrire l'histoire des femmes et du genre, op. cit. 
La séquence du débat sur la parité est symptomatique de cette évolution. Dès 1989, à la faveur des commémorations du bicentenaire de la Révolution française, se fait jour un questionnement commun aux différents pôles académique, associatif et institutionnel de l'espace de la cause des femmes. Alors que la revendication de la parité en politique apparaît dans le pôle associatif et institutionnel, se développe parallèlement une histoire qui porte un regard critique sur les rapports entre femmes et pouvoir politique. Par exemple, les travaux de Dominique Godineau (Citoyennes tricoteuses. Les femmes du peuple à Paris pendant la Révolution française), Catherine Marand-Fouquet (La Femme au temps de la Révolution) et Geneviève Fraisse (Muse de la raison), parus entre 1988 et 1989, réévaluent le rôle joué par les femmes dans la Révolution et, ce faisant, soulèvent la question du lien entre démocratie et exclusion des femmes. Il en est de même avec les travaux sur l'Ancien Régime présentés lors du colloque organisé par Éliane Viennot et Danielle Haase Dubosc, intitulé Femmes et pouvoir sous l'Ancien Régime. Ce questionnement commun aux différentes composantes de l'espace de la cause des femmes va se nourrir du dialogue entre chercheuses, fémocrates et militantes. Laure Bereni explique ainsi comment la notion de «démocratie paritaire » a vu le jour à la suite d'une rencontre entre Claudette Apprill, administratrice du « comité chargé de l'égalité entre les femmes et les hommes » (comité Égalité) du Conseil de l'Europe jusqu'au début des années 1990, et Élisabeth Sledziewski, chercheuse articulant philosophie et histoire politique dans ses travaux sur le «sujet féminin»sous la Révolution. Ces dernières se rencontrent lors du colloque «Les femmes et la Révolution française» organisé par l'historienne Marie-France Brive, à Toulouse, auquel Claudette Apprill se rend « à titre privé » et pendant lequel elle est «"scandalisée" par les "termes" dans lesquels les femmes ont été historiquement exclues de la démocratie $»^{70}$. Claudette Apprill et Élisabeth Sledziewski commencent alors ensemble un travail de réflexion sur le rapport des femmes à la démocratie, et plus précisément autour de la notion de «démocratie paritaire ». Cet épisode illustre le renforcement du jeu d'influence réciproque entre recherches historiques et revendications politiques à partir du milieu dans années 1990: d'un côté, les militantes s'appuient sur les « leçons de l'histoire ${ }^{71}$ et de l'autre, les historiennes des femmes, dans une

70. Laure Bereni, « De la cause à la loi : les mobilisations pour la parité politique en France (1992-2000) », thèse de doctorat en science politique, Paris, Université Paris 1 Panthéon Sorbonne, 2007, p. 117.

71. C'est le cas notamment dans l'ouvrage Au pouvoir citoyennes! de Anne Le Gall, Françoise Gaspard et Claude Servan-Schreiber. 
«dialectique présent-passé propre à tout domaine historique $»^{72}$, adaptent leurs objets et questionnements scientifiques à l'actualité.

Pendant les années 1990, les historiennes s'engagent aussi plus fortement dans la diffusion du passé des femmes en dehors des cercles académiques, dans un contexte national et international plus favorable. On assiste autour de 1995 à un réveil du féminisme en France avec la Conférence mondiale sur les femmes de Pékin, la manifestation de rue du 25 novembre 1995 pour les droits des femmes et l'émergence des débats sur la parité. Une nouvelle génération de femmes s'engage dans des groupes féministes, et manifeste alors un intérêt pour le passé des femmes. Les médias attisent et répondent à cette attente en donnant la parole aux chercheuses en études féministes: «Un certain nombre de spécialistes des questions "femmes" et "genre" accèdent aux maisons d'édition grand public, aux journaux, aux télévisions et gagnent ainsi un statut particulier, plus gratifiant en général que celui qu'elles/ils ont dans le monde académique ${ }^{73}$. À la fin des années 1990 des ouvrages de synthèse et des manuels de vulgarisation visant la formation des étudiantes et une diffusion de l'histoire des femmes hors du champ académique commencent à paraître ${ }^{74}$. Ce travail de valorisation extra-académique de la recherche n'est pas à proprement parler une démarche mémorielle mais elle fournit aux militantes les ressources et la dynamique sur lesquelles fonder leurs tentatives d'élaboration d'une mémoire collective.

Les rapports entre les différents pôles de l'espace de la cause des femmes ne se résument pas à ces seules influences réciproques diffuses : au moment du bicentenaire, les historiennes des femmes s'engagent également dans des actions plus volontaristes visant à l'élaboration d'une mémoire féministe et à sa diffusion auprès du grand public. Une des premières actions à vocation mémorielle, portée par l'historienne Catherine Marand-Fouquet, est une campagne ${ }^{75}$ en faveur de la panthéonisation d'Olympe de Gouges, figure de la lutte

72. Françoise Thébaud évoque, à titre d'exemple, l'émergence d'une histoire européenne encore balbutiante suscitée par les transformations et les difficultés de l'Europe communautaire. Voir F. Thébaud, Écrire l'histoire des femmes et du genre, op. cit., p. 177.

73. Christine Bard, «Recherche et militantisme (France, 1995-2002)», in Christine Bard, Christian Baudelot et Janine Mossuz-Lavau, Quand les femmes s'en mêlent. Genre et pouvoir, Paris, Éd. de La Martinière, 2004, p. 266.

74. Par exemple, Les Femmes actrices de l'Histoire. France, 1789-1945, de Yannick Ripa ; Les Femmes dans la société française au $20^{e}$ siècle, de Christine Bard; Histoire du féminisme, de Michèle Riot-Sarcey ; Les Femmes dans la société française, $16^{e}-18^{e}$ siècle, de Dominique Godineau; Les Femmes à l'époque moderne $\left(X_{V I}{ }^{e}\right.$ XVIII ${ }^{e}$ siècles, de Scarlett Beauvalet-Boutouyrie; ou Histoire des femmes en France, XIX ${ }^{e}-X X^{e}$ siècles, de Michelle Zancarini-Fournel.

75. Cette campagne est lancée le $1^{\mathrm{er}}$ septembre 1989, lorsque l'historienne Catherine Marand-Fouquet, qui vient de publier son ouvrage sur La Femme au temps de la Révolution, écrit une lettre au Monde dans laquelle elle demande l'entrée d'Olympe de Gouges au Panthéon. 
pour les droits des femmes pendant la Révolution et rédactrice d'une Déclaration des droits de la femme et de la citoyenne. Le coup de projecteur mis sur les héros et héroïnes de la Révolution française au moment du bicentenaire est favorable au retour dans l'imaginaire féministe de figures comme celles d'Olympe de Gouges ou Théroigne de Méricourt ${ }^{76}$. Très vite, la revendication de panthéonisation trouve un écho chez des militantes du pôle associatif $^{77}$ : Gisèle Halimi conseille même à Catherine Marand-Fouquet d'aller plus loin en associant à sa demande celles des panthéonisations de Marie Curie et de la résistante Berty Albrecht $^{78}$. Malgré la mobilisation et l'envoi de lettres au président de la République, François Mitterrand, la revendication reste lettre morte : la figure d'Olympe de Gouges n'est pas assez consensuelle et selon François Mitterrand, il est de toute façon trop tard pour 1989. Le bicentenaire de la mort d'Olympe de Gouges, le 3 novembre 1993, en plein essor de la lutte pour la parité, est l'occasion, de réactiver cette revendication mémorielle : une centaine de féministes (notamment les militantes du réseau Femmes pour la parité et un collectif de féministes marseillaises constitué autour de Catherine Marand-Fouquet) manifestent, le 6 novembre 1993, devant les grilles du Panthéon et déposent une gerbe en souvenir de l'icône féministe révolutionnaire. «Les manifestantes demandent le transfert de ses cendres et de celles d'autres femmes illustres $\gg^{79}$. Les campagnes de panthéonisation sont régulièrement relancées au cours des années 2000, soutenues par d'autres historiennes comme Christine Bard. Les figures de femmes concernées varient selon les collectifs et selon la période. Elles se soldent par l'entrée au Panthéon de Marie Curie en 1995, puis par la décision de François Hollande, en 2015, d'y accueillir, aux côtés de deux hommes (parité oblige), deux résistantes : Germaine Tillion et Geneviève de Gaulle-Anthonioz, deux figures rarement mises en avant par les féministes.

Finalement, c'est à la fois l'institutionnalisation de l'histoire des femmes et ses limites qui permettent une nouvelle convergence entre histoire et mémoire au sein de la cause des femmes : les historiennes endossent une posture militante pour diffuser la cause des femmes dans les institutions et, corrélativement, elles utilisent leurs positions et les ressources offertes par l'académie pour alimenter les pratiques mémorielles des militantes de la cause des

76. Élisabeth Roudinesco, historienne, alors directrice de recherche à l'Université Paris 7 lui consacre un ouvrage.

77. Christine Bard précise que, chez les chercheuses féministes, «toutes ne souhaitaient pas du tout voir "leur" Olympe au Panthéon ». C'est le cas par exemple, de Marie-France Brive et le groupe Simone de l'Université Toulouse Le Mirail. Voir C. Bard, «Les usages politiques de l'histoire des femmes », op. cit.

78. Ibid.

79. L. Bereni, « De la cause à la loi... », op. cit., p.204. 
femmes. La création du Centre des archives du féminisme et de l'association Archives du féminisme est un exemple de l'usage stratégique par une historienne de sa position académique pour impulser des actions à vocation mémorielle.

En s'appuyant sur les ressources que lui offre sa position académique, Christine Bard développe un réseau d'initiatives visant à la conservation des traces laissées par les groupes ou militantes féministes (Centre des archives du féminisme d'Angers), à la valorisation de ces traces (association Archives du féminisme) et à la diffusion de l'histoire des femmes auprès du grand public (exposition sur les grilles du Panthéon, campagnes pour les panthéonisations, musées d'histoire des femmes, etc.).

La conservation des traces constitue un enjeu mémoriel important : très tôt des féministes sont conscientes qu'un des obstacles à la transmission d'une mémoire féministe est la perte des traces laissées par les collectifs. Ainsi, des figures de la «première vague », telles Eliska Vincent, Marbel, Marguerite Durand ou Marie-Louise Bouglé ont fait de la conservation des archives le cœur de leur activité militante. Christine Bard, qui a consacré sa thèse aux féminismes des années 1914 à 1940 ${ }^{80}$, inspirée par le travail réalisé par ces pionnières, décide de s'engager dans la création du Centre des archives du féminisme (CAF). Recrutée en 1995 comme maîtresse de conférences en histoire contemporaine à l'Université d'Angers, où existe une formation en archivistique, elle se familiarise avec les principes et les techniques qui régissent la préservation des archives. La bibliothèque d'Angers possédant déjà des fonds spécialisés est donc en mesure d'apporter son expertise. En juillet 2000, Christine Bard est nommée à l'Institut universitaire de France et obtient une décharge de cours ainsi qu'un budget de recherche conséquent pendant cinq ans, qui lui permet de s'engager dans un projet comme le CAF. Le centre d'Angers existe à la fois comme en tant qu'association Archives du féminisme depuis 2000 et comme structure universitaire, le CAF inauguré en 2001, cette dualité de structures permettant aux féministes d'exercer un certain contrôle sur leurs archives et d'assurer au centre une pérennité et des moyens financiers et humains dont disposent rarement les associations féministes.

80. Christine Bard, Les Filles de Marianne. Histoire des féminismes 1914-1940, Paris, Fayard, 1995. 
Après une première phase de proximité entre histoire des femmes et sphère militante, le souci des historiennes de s'intégrer dans la discipline et donc de répondre aux exigences académiques, les détournent de l'enjeu mémoriel et de l'objet d'études mémoire. Ce sont finalement les résistances de la discipline historique qui posent des limites à l'institutionnalisation de l'histoire des femmes et qui conduisent ses tenantes à renouer avec une posture plus militante, que ce soit dans les institutions elles-mêmes, ou dans la société en général. À partir des années 1990, une nouvelle génération d'historiennes s'investit dans des actions mémorielles en collaboration avec la sphère militante et étatique.

Alors que dans un premier temps, l'entrée de l'histoire des femmes dans le champ académique pouvait apparaître comme un risque pour l'unité et l'autonomie de la cause des femmes, son institutionnalisation a participé finalement à la consolidation d'un espace de cette cause, en la stabilisant, en la confortant, et ce de façon relativement homogène : autour de la mémoire, se développent un ensemble de pratiques communes et un système de relations sociales entre les différentes sphères associatives, académiques et politiques. En suggérant des interprétations du passé appropriables par les militantes, en travaillant à la conservation des traces féministes, en offrant des positions de pouvoir permettant la diffusion d'un récit du passé concurrent à la doxa ambiante, l'institutionnalisation de l'histoire des femmes a été ainsi pourvoyeuse en ressources pour construire une mémoire collective féministe. 